\title{
Charge ordering in Ir dimers in the ground state of $\mathrm{Ba}_{5} \mathrm{AlIr}_{2} \mathrm{O}_{11}$
}

\author{
Vamshi M. Katukuri $\odot,{ }^{1, *}$ Xingye Lu $\odot,{ }^{2,3, \dagger}$ D. E. McNally, ${ }^{2}$ Marcus Dantz, ${ }^{2}$ Vladimir N. Strocov,${ }^{2}$ M. Moretti Sala,,${ }^{4,5}$ \\ M. H. Upton, ${ }^{6}$ J. Terzic, ${ }^{7}$ G. Cao, ${ }^{7}$ Oleg V. Yazyev, ${ }^{1}$ and Thorsten Schmitt ${ }^{2,}$ \\ ${ }^{1}$ Institute of Physics, École Polytechnique Fédérale de Lausanne (EPFL), CH-1015 Lausanne, Switzerland \\ ${ }^{2}$ Swiss Light Source, Photon Science Division, Paul Scherrer Institut, CH-5232 Villigen PSI, Switzerland \\ ${ }^{3}$ Center for Advanced Quantum Studies and Department of Physics, Beijing Normal University, Beijing 100875, China \\ ${ }^{4}$ European Synchrotron Radiation Facility, BP 220, F-38043 Grenoble Cedex, France \\ ${ }^{5}$ Dipartimento di Fisica, Politecnico di Milano, piazza Leonardo da Vinci 32, I-20133 Milano, Italy \\ ${ }^{6}$ Advanced Photon Source, Argonne National Laboratory, Argonne, Illinois 60439, USA \\ ${ }^{7}$ Department of Physics, University of Colorado at Boulder, Boulder, Colorado 80309, USA
}

(Received 19 March 2021; revised 17 December 2021; accepted 23 December 2021; published 7 February 2022)

\begin{abstract}
It has been well established experimentally that the interplay of electronic correlations and spin-orbit interactions in $\operatorname{Ir}^{4+}$ and $\operatorname{Ir}^{5+}$ oxides results in insulating $J_{\text {eff }}=1 / 2$ and $J_{\text {eff }}=0$ ground states, respectively. However, in compounds where the structural dimerization of iridium ions is favorable, the direct Ir $d$ - $d$ hybridization can be significant and takes a key role. Here, we investigate the effects of direct Ir $d$ - $d$ hybridization in comparison with electronic correlations and spin-orbit coupling in $\mathrm{Ba}_{5} \mathrm{AlIr}_{2} \mathrm{O}_{11}$, a compound with Ir dimers. Using a combination of $a b$ initio many-body wave-function quantum chemistry calculations and resonant inelastic x-ray scattering experiments, we elucidate the electronic structure of $\mathrm{Ba}_{5} \mathrm{AlIr}_{2} \mathrm{O}_{11}$. We find excellent agreement between the calculated and the measured spin-orbit excitations. Contrary to expectations, the analysis of the many-body wave function shows that the two $\mathrm{Ir}_{\left(\mathrm{Ir}^{4+}\right.}$ and $\left.\mathrm{Ir}^{5+}\right)$ ions in the $\mathrm{Ir}_{2} \mathrm{O}_{9}$ dimer unit in this compound preserve their local $J_{\text {eff }}$ character close to $1 / 2$ and 0 , respectively. The local point group symmetry at each of the Ir ions plays an important role, significantly limiting the direct $d$ - $d$ hybridization. Our results emphasize that minute details in the local crystal field environment can lead to dramatic differences in the electronic states in iridates and $5 d$ oxides in general.
\end{abstract}

DOI: 10.1103/PhysRevB.105.075114

\section{INTRODUCTION}

Dimerization or clustering of transition metal (TM) atoms is observed in many TM compounds, e.g., in vanadium oxides [1,2], and titanates [3], when the $t_{2 g}$ orbitals of the TM $d$ manifold form partially filled singlet states of dimers akin to the Peierls state in one dimension [4]. In these systems, the TM ions tend to have a strong direct (intradimer) $d-d$ overlap that results in molecularlike orbitals with appreciable bonding-antibonding splitting. Consequently, the local electronic structure depends on the intradimer hopping integral $\left(t_{d}\right)$, intra-atomic Hund's coupling $\left(J_{H}\right)$, and interatomic $(U)$ Coulomb interactions and electron filling of the orbitals localized at TM clusters. Alternatively, dimerization of TM ions can also be favorable from crystallographic considerations, particularly in compounds with heavy TM ions, e.g., $5 d$ ions, where the $d$ orbitals are more spread out. A number of dimerized or cluster $4 d$ and $5 d$ compounds [5-8] with intriguing properties have been synthesized recently. Novel physical phenomena have been observed in these compounds, e.g.,

\footnotetext{
*Present address: Max Planck Institute for Solid State Research, Heisneberstrasse 1, 70569, Stuttgart Germany; V.Katukuri@fkf.mpg.de

†luxy@bnu.edu.cn

†thorsten.schmitt@psi.ch
}

the inelastic x-ray scattering analog of Young's double-slit experiment has been realized in $\mathrm{Ba}_{3} \mathrm{CeIr}_{2} \mathrm{O}_{9}$ [9], where the molecular orbital formation within the Ir dimers is crucial. In lacunar spinels $\mathrm{Ga}_{4} X_{8}(M=\mathrm{Nb}, \mathrm{Mo}$, Ta, and $\mathrm{W}$ and $X=\mathrm{S}$, $\mathrm{Se}$, and Te), spin-orbit coupled molecular $J_{\text {eff }}$ states $[10,11]$ and topological superconductivity [12] have been proposed where molecular orbital formation within the tetrahedral cluster of $M$ ions is the key.

The interplay of intersite electron hopping $(t), J_{H}, U$, and the strong atomic spin-orbit coupling (SOC) in $5 d$ and in some $4 d$ compounds result in intriguing $J_{\text {eff }}$ physics [13-17]. For instance, in compounds with an $\operatorname{Ir}^{4+}\left(d^{5}\right)$ configuration in an octahedral environment, e.g., in $\mathrm{Sr}_{2} \mathrm{IrO}_{4}[13,14]$, the strong SOC leads to completely filled $J_{\text {eff }}=3 / 2$ and halffilled $J_{\text {eff }}=1 / 2$ levels. Similarly, in $\mathrm{Ba}_{2} \mathrm{YIrO}_{6}$ and $\mathrm{NaIrO}_{3}$, the $\mathrm{Ir}^{5+}$ ions realize completely filled $J_{\text {eff }}=3 / 2$ and empty $J_{\text {eff }}=1 / 2$ submanifolds $[18,19]$, resulting in a nonmagnetic $J_{\text {eff }}=0$ ground state [20]. In dimerized systems, $t_{d}$ can be much larger and successively may play a dominant role compared to other local interactions, which could result in the breakdown or a significant modification of the $J_{\text {eff }}$ physics. Thus, it is crucial to identify the role of these multiple interactions in Ir dimer systems to gain a better understanding of the electronic and magnetic properties of these materials.

In this paper, we illustrate how subtle crystal structure details are extremely important to precisely understand the electronic structure of $5 d$ compounds where structural 
dimerization or clustering is prevalent. Using state-of-the-art $a b$ initio many-body electronic structure methods in combination with high-resolution resonant inelastic $\mathrm{x}$-ray scattering (RIXS) experiments, we present a detailed analysis of the electronic structure of $\operatorname{Ir}_{2} \mathrm{O}_{9}$ dimers in $\mathrm{Ba}_{5} \mathrm{AlIr}_{2} \mathrm{O}_{11}$ and unravel the nature of the electronic ground and excited states of $\mathrm{Ba}_{5} \mathrm{AlIr}_{2} \mathrm{O}_{11}$. While we find an excellent agreement between the RIXS spectra and the calculated excitations, analysis of the many-body wave functions reveals a nearly complete charge separation $-\mathrm{Ir}^{4+}$ and $\mathrm{Ir}^{5+}$ - within the dimers in the ground state, in contrast to an earlier report describing the formation of molecular orbitals in $\mathrm{Ba}_{5} \mathrm{AlIr}_{2} \mathrm{O}_{11}$ [21]. The strong SOC of the $\mathrm{Ir}^{4+}$ and $\mathrm{Ir}^{5+}$ ions results in $J_{\text {eff }}=1 / 2$ and $J_{\text {eff }}=0$ local configurations, respectively, and thus we conclude that a localized $J_{\text {eff }}$ picture is more appropriate in $\mathrm{Ba}_{5} \mathrm{AlIr}_{2} \mathrm{O}_{11}$.

$\mathrm{Ba}_{5} \mathrm{AlIr}_{2} \mathrm{O}_{11}$ contains dimers composed of crystallographically inequivalent $\mathrm{Ir}$ cations encaged in face-sharing $\mathrm{O}_{6}$ octahedra [22,23] [see Figs. 3(a) and 3(b), and Supplemental Material (SM) Fig. S1 [24]]. At $210 \mathrm{~K}$, a lattice distortion is believed to lower the symmetry of the crystal and enhance the charge disproportionation leading to charge ordering that corresponds to $\mathrm{Ir}^{4+}$ and $\mathrm{Ir}^{5+}$ valence configurations [23]. However, an analysis of the previously measured RIXS spectrum of $\mathrm{Ba}_{5} \mathrm{AlIr}_{2} \mathrm{O}_{11}$ using density functional theory and model Hamiltonian calculations [21] has proposed the formation of hybridized dimer orbitals, debunking the charge disproportionation phenomenon. Nevertheless, given the complex low-symmetry crystal environment and the interplay of spin and orbital degrees of freedom in $\mathrm{Ba}_{5} \mathrm{AlIr}_{2} \mathrm{O}_{11}$, it is unclear if the dimer orbitals are actually realized in the ground state.

\section{RESULTS}

The RIXS spectra shown in Fig. 1 were measured on single crystals grown by the flux method [23] at the ID20 beamline of the European Synchrotron Radiation Facility (ESRF) with $\sim 25 \mathrm{meV}$ resolution [25] and the 27-ID-B beamline with $\sim 30 \mathrm{meV}$ resolution at the Advanced Photon Source (APS), with $\pi$ polarization at a scattering angle close to $2 \theta=90^{\circ}$. The incident-energy dependence of RIXS spectra across the Ir $L_{3}$ edge $\left(E_{i}=11.215 \mathrm{keV}\right)$ at the zone center $Q=(23.5,0,2.5)$ is shown in Fig. 1(a). While the same $E_{i}$ as determined from previous measurements on iridates such as $\mathrm{Sr}_{2} \mathrm{IrO}_{4}$ and $\mathrm{Ba}_{2} \mathrm{YIrO}_{6}[20,26]$ was chosen, we find that the maximum of the resonance is not at $E_{i}$ in $\mathrm{Ba}_{5} \mathrm{AlIr}_{2} \mathrm{O}_{11}$ as the precise crystal field (CF) environment around Ir ions and the mixing of the valence states influences the resonance energy. However, we see that the energies of the modes remain unchanged in a broad range around $E_{i}$.

The features marked by $A-K$ in Fig. 1(b) are incidentenergy independent Raman modes as shown in Fig. 1(a). These modes correspond to intrinsic electronic transitions between various occupied and unoccupied states, and therefore provide direct information about the low-energy electronic structure. To resolve all the Raman modes and determine the low-energy electronic structure, we show in Figs. 1(b) and 1 (c) the high statistic energy spectra collected at $E_{i}$ [white dashed line in Fig. 1(a)]. In Fig. 1(b), several sharp Raman modes below $1 \mathrm{eV}$ and a broad peak at $1.2 \mathrm{eV}$, named $A-K$,

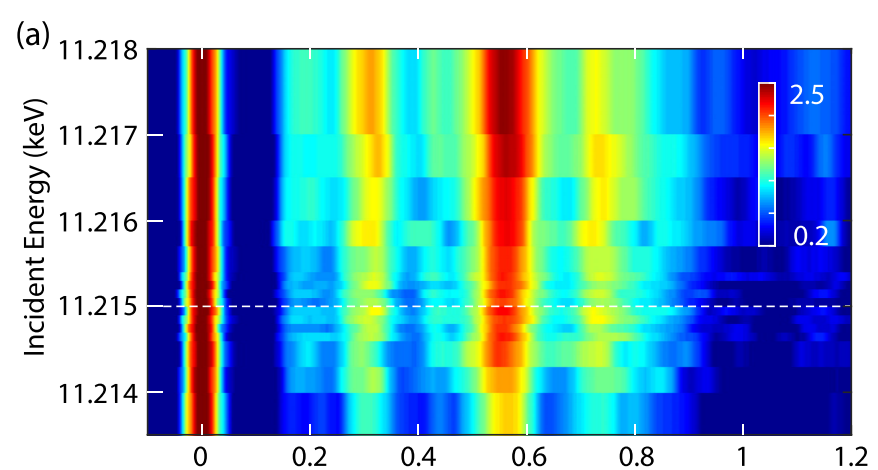

(b)

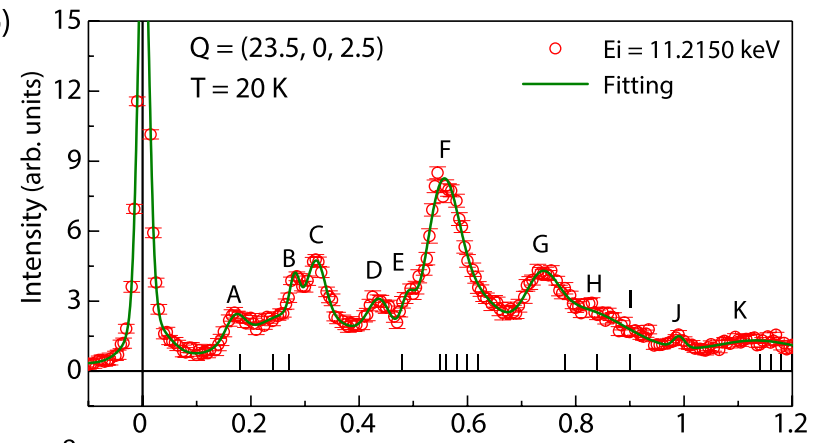

(c)

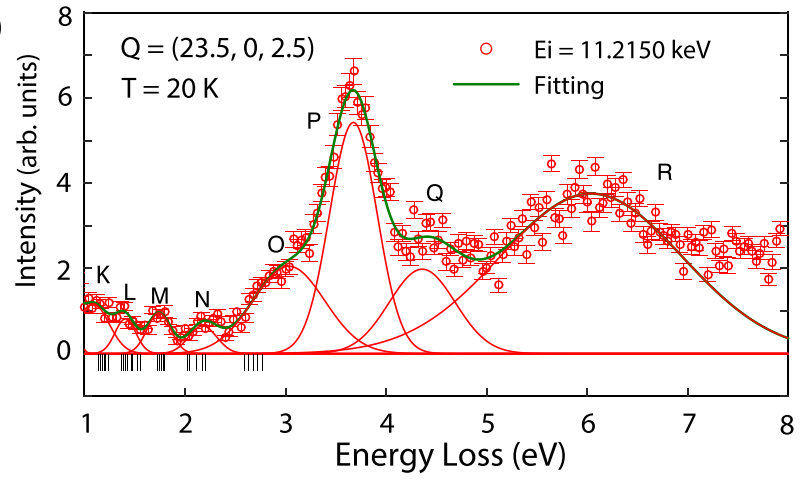

FIG. 1. (a) Incident-energy dependence of elementary excitations below $1.2 \mathrm{eV}$ for $\mathrm{Ba}_{5} \mathrm{AlIr}_{2} \mathrm{O}_{11}$ measured around the $\mathrm{Ir} L_{3}$ edge with $Q=(23.5,0,2.5)$ at $20 \mathrm{~K}$. (b) RIXS spectra (below $1.2 \mathrm{eV}$ ) measured at $E_{i}=11.215 \mathrm{keV}$ [marked as a white dashed line in (a)]. (c) High-energy excitations (1-8 eV) measured with the same setup as that for (b). The green line is a multi-Gaussian fitting of the raw data in red open circles (with error bars).

are determined by fitting the spectra using multiple Gaussians. The sum of the fitting curves is shown as a green solid curve. In Fig. 1(c), higher-energy excitations up to $8 \mathrm{eV}$ are shown. This spectrum is decomposed into several peaks and interestingly, these modes show very little momentum dependence (see SM Fig. S6 [24]), indicating that all of them correspond to local spin-orbital $(d-d)$ excitations and are reflecting the low-energy electronic structure.

We now turn to the RIXS results measured using the $\mathrm{O}$ $K$ edge (Fig. 2 and Fig. S2 in SM [24]) carried out at the ADRESS beamline of the Swiss Light Source at the Paul Scherrer Institut, with $\sim 70 \mathrm{meV}$ energy resolution for both $\sigma$ and $\pi$ polarizations at a scattering angle of $2 \theta=130^{\circ}[27,28]$ (see SM Fig. S1). With the presence of strong hybridization between the $\mathrm{O} 2 p$ orbitals and Ir $5 d$ orbitals, O $K$ RIXS is sensitive to various elementary excitations of iridates [29]. Figures 2(a) and 2(b) are RIXS maps collected at the $\mathrm{O} K$ edge 


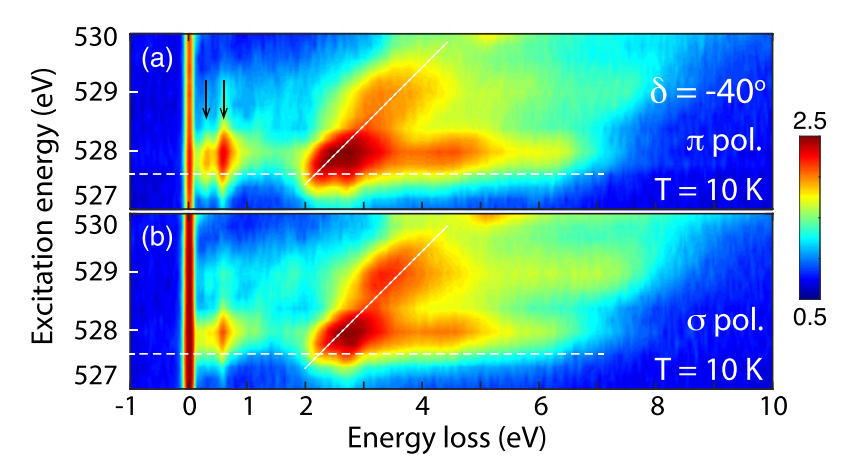

FIG. 2. RIXS results of $\mathrm{Ba}_{5} \mathrm{AlIr}_{2} \mathrm{O}_{11}$ as measured at the $\mathrm{O} K$ edge. (a), (b) Energy dependence of RIXS spectra for $\mathrm{Ba}_{5} \mathrm{AlIr}_{2} \mathrm{O}_{11}$ taken around the $\mathrm{O} K$ edge with $\delta=-40^{\circ}[Q=(0.93,0,0.65)]$.

with $\pi$ and $\sigma$ polarizations at $25^{\circ}$ grazing incidence. Besides the sharp spin-orbital excitations $(E \approx 0.26,0.57 \mathrm{eV})$ below $1 \mathrm{eV}$ consistent with those measured with the Ir $L_{3}$ edge, two high-energy excitations at $E \approx 2.26$ and $2.71 \mathrm{eV}$ have also been observed. Note the RIXS maps in Fig. 2 contain substantial fluorescence which is absent in the results collected at the $\operatorname{Ir} L_{3}$ edge (Fig. 1), indicating a complex Ir $5 d$-O $2 p$ hybridization between the energy bands of the oxygen ions. In addition, a significant polarization dependence of the excitations has also been observed, which we attribute to the overlap between light polarization (electric field $\mathbf{E}$ ) and the different $\mathrm{O} 2 p$ orbitals hybridized with different Ir $5 d$ orbitals (for details, see SM [24]).

To decipher the nature of the rich excitation spectrum observed in the RIXS spectra and to examine the formation of dimer orbitals in $\mathrm{Ba}_{5} \mathrm{AlIr}_{2} \mathrm{O}_{11}$, we performed many-body $a b$ initio cluster-in-embedding quantum chemistry (QC) calculations, starting from the crystal structure reported in Refs. $[22,23]$. These are based on the construction of the exact wave function for a cluster of atoms, embedded in a potential that represents the solid-state environment, using complete active space self-consistent field (CASSCF) and multireference perturbation methods [30]. The calculations were performed on a cluster containing one $\mathrm{Ir}_{2} \mathrm{O}_{9}$ dimer unit, two neighboring $\mathrm{AlO}_{4}$ tetrahedra, and the surrounding $15 \mathrm{Ba}^{2+}$ ions. The ORCA quantum chemistry program [31] was used for all calculations (see SM [24] for all the computational details, which includes Refs. [29,31-36]).

The relative energies of the multiplet structure of the $\operatorname{Ir}_{2} \mathrm{O}_{9}$ dimer unit obtained from CASSCF + NEVPT2 $(N$-electron valence perturbation theory) [37] calculations are shown in Table I. An active space of nine electrons in six orbitals (three $t_{2 g}$ orbitals on each iridium) was considered in the CASSCF calculation which sufficiently captures the important static correlations (i.e., near degeneracies) in $\mathrm{Ba}_{5} \mathrm{AlIr}_{2} \mathrm{O}_{11}$. In the NEVPT2 calculation, the correlations involving all the neighboring occupied oxygen $2 p$ and iridium $5 s, 5 p$ orbitals as well as all the unoccupied orbitals are accounted for, accurately describing the $\mathrm{O} 2 p$ to Ir $d$ charge transfer effects and other dynamic correlation effects. It is important to note that the intrasite (Hund's coupling $J_{H}$ ) and intersite $(U)$ Coulomb interactions and the hybridization between different orbitals are included in the calculation, accurate within the basis-set limit.
TABLE I. Relative energies (eV) of the excitation levels obtained from CASSCF+NEVPT2 calculations. The first column contains the nonrelativistic multiplet structure, and the multiplet symbols on the left correspond to the octahedral $\left(O_{h}\right)$ symmetry. The degeneracy of the states is split in $\mathrm{Ba}_{5} \mathrm{AlIr}_{2} \mathrm{O}_{11}$ due to the lowered symmetry in the two octahedra due to the anisotropic crystal fields (see text). The spin-orbit coupled multiplet structure is shown in the second column. Note that each state is doubly degenerate (Kramers doublet). The corresponding peaks in the RIXS data in Fig. 1(a) are shown in column 3 .

\begin{tabular}{lccc}
\hline \hline \multicolumn{2}{c}{ CASSCF+NEVPT2 } & + SOC $(\times 2)$ & Ir $L$-edge RIXS \\
\hline${ }^{4} A_{1}$ & 0.00 & 0.00 & 0.00 \\
${ }^{2} T_{1}$ & $0.03,0.08,0.10$ & 0.18 & $0.18(A)$ \\
${ }^{2} A_{1}$ & 0.14 & $0.24,0.27$ & $0.28(B) 0.33(C)$ \\
${ }^{4} T_{1}$ & $0.16,0.17,0.17$ & & $0.44(D)$ \\
${ }^{2} E_{1}$ & $0.18,0.23$ & $0.48,0.62$ & $0.50(E)$ \\
${ }^{4} E_{1}$ & $0.25,0.28$ & $0.55,0.56,0.58$ & $0.56(F)$ \\
${ }^{2} T_{2}$ & $0.77,0.80,0.94$ & $0.60,0.78$ & $0.75(G)$ \\
${ }^{4} T_{2}$ & $0.84,0.86,0.95$ & $0.84,0.90$ & $0.82(H)$ \\
${ }^{2} T_{3}$ & $0.86,0.86,0.90$ & $1.14-1.20(4)$ & $0.98(I), 1.00(J)$ \\
${ }^{2} A_{2}$ & 0.91 & $1.21,1.24$ & $1.20(K)$ \\
${ }^{2} E_{2}$ & $1.00,1.01$ & $1.37-1.43(4)$ & $1.4(L)$ \\
${ }^{2} A_{3}$ & 1.03 & 1.47 & \\
${ }^{2} T_{4}$ & $1.10,1.10,1.12$ & $1.48,1.53,1.56$ & $1.77(M)$ \\
${ }^{2} E_{3}$ & $1.60,1.63$ & $1.73-1.80(5)$ & $2.17(N)$ \\
${ }^{2} T_{5}$ & $1.68,1.78,1.81$ & $2.02,2.04$ & $2.70(O)$ \\
\multicolumn{4}{c}{$2.11,2.17,2.20$} \\
\hline
\end{tabular}

The lowest nine quartet $\left(s=\frac{3}{2}\right)$ and 24 doublet $\left(s=\frac{1}{2}\right)$ scalar relativistic states (first column in Table I) are first computed and then are allowed to admix via the SOC, resulting in 84 states (see the second column of Table I). It can be seen that the excitation energies obtained from CASSCF + NEVPT2 + SOC calculations are in excellent agreement with the peaks observed in RIXS experiments, except for peak $D$. This peak is related to the electron-hole exciton which is also observed in other iridate materials such as $\mathrm{Sr}_{2} \mathrm{IrO}_{4}[29,38,39]$ and $\mathrm{Na}_{2} \mathrm{IrO}_{3}$ [40]. Such excitations are not considered in the current QC calculations [41]. Further, our calculations reveal excitations from the $t_{2 g}$ to $e_{g}$ manifold starting at $3.4 \mathrm{eV}$ which correspond to RIXS peaks $P$ and $Q$.

To elucidate the origin of these excitations, we first analyze the scalar-relativistic multiplet structure. When the two iridium ions in the dimer unit are in a cubic environment $\left(O_{h}\right.$ symmetry), the low-energy multiplet structure is a result of the interaction of the ground state ${ }^{2} T_{1 g}$ multiplet of the $\mathrm{Ir}^{4+}$ ion [42] and the ${ }^{3} T_{1 g}$ ground state term of the $\mathrm{Ir}^{5+}$ ion. In addition, the lowest ${ }^{1} T_{1 g}$ and ${ }^{1} E_{1 g}$ [20] singlet states contribute significantly to the low-energy spin-orbit excitations [20]. The resulting spectrum contains ${ }^{4} T_{1 g},{ }^{4} T_{2 g},{ }^{4} A_{1 g},{ }^{4} E_{1 g}$ quartets and 11 doublet terms, ${ }^{2} T_{1 g, 2 g, 3 g, 4 g, 5 g},{ }^{2} A_{1 g, 2 g, 3 g},{ }^{2} E_{1 g, 2 g, 3 g}$ [43]. However, in $\mathrm{Ba}_{5} \mathrm{AlIr}_{2} \mathrm{O}_{11}$ the Ir ions are enclosed in distorted octahedra, resulting in low-symmetry CFs and splitting of the $t_{2 g}$ levels at each Ir ion [44]. Further, the small Ir-Ir intradimer distance of $2.73 \AA$ in $\mathrm{Ba}_{5} \mathrm{AlIr}_{2} \mathrm{O}_{11}(2.698 \AA$ in elemental iridium) may result in a direct overlap of the $\operatorname{Ir} d$ orbitals 


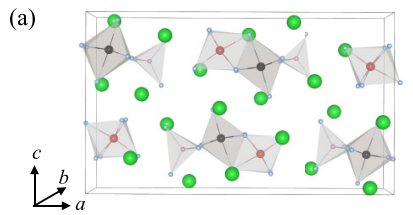

(b) $\square \mathrm{Ba}$

(c)

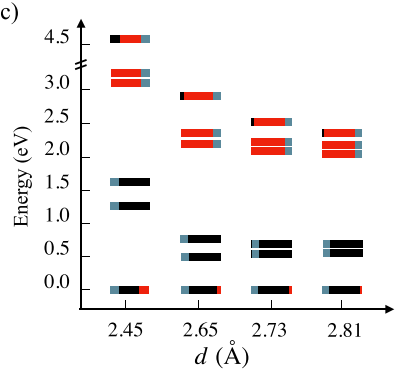

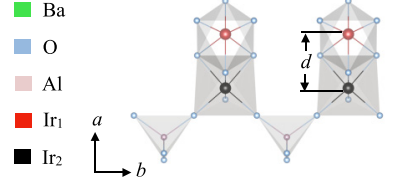

(d)

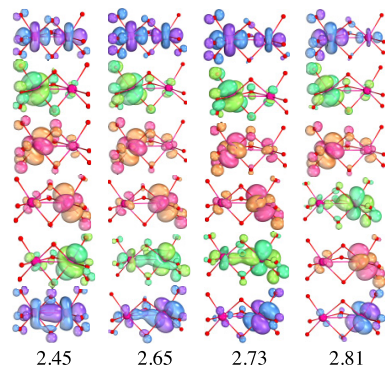

FIG. 3. Crystal structure of $\mathrm{Ba}_{5} \mathrm{AlIr}_{2} \mathrm{O}_{11}$ : (a) Unit cell, (b) $\mathrm{Ir}_{2} \mathrm{O}_{9}$ dimer units connected along the $b$ axis, and $d$ is the intradimer $\operatorname{Ir}_{1}-\mathrm{Ir}_{2}$ distance. (c) Orbital (relative) energy-level diagram of the six $t_{2 g}$ orbitals, shown in (d), for different $d$, where the lowest-energy orbital is set to zero. The lengths of the red, black, and blue colors of each level are proportional to the percentage contributions from $\mathrm{Ir}_{1}, \mathrm{Ir}_{2}$, and $\mathrm{O}$ ions, respectively.

and the formation of bonding and antibonding states [45]. Consequentially, the multiplet degeneracies in the spectrum are split.

To understand the formation of bonding and antibonding dimer orbitals in $\mathrm{Ba}_{5} \mathrm{AlIr}_{2} \mathrm{O}_{11}$, we plot the evolution of orbital energies as a function of $\operatorname{Ir}_{1}-\mathrm{Ir}_{2}$ intradimer distance $(d)$ in Fig. 3(c). The six levels for each $d$ correspond to the CASSCF canonical orbital [46] energies of the six $t_{2 g}$-like orbitals in the $\mathrm{Ir}_{2} \mathrm{O}_{9}$ dimer unit. The color variations of the energy levels represent the orbital compositions [47] from $\mathrm{Ir}_{1}, \mathrm{Ir}_{2}$, and $\mathrm{O}$ ions. Interestingly, for $d \geqslant 2.73 \AA$, we find $20 \%$ and $13 \%$ hybridization for $\operatorname{Ir}_{1} 5 d-\mathrm{O} 2 p$ and $\operatorname{Ir}_{2} 5 d-\mathrm{O} 2 p$, respectively, while there is negligible direct $\operatorname{Ir}_{1}-\mathrm{Ir}_{2} d$-orbital hybridization. The $a_{1 g}$ orbital of $\operatorname{Ir}_{1}$ contains a $4.5 \%$ contribution from the $a_{1 g}$ orbital of $\operatorname{Ir}_{2}$ and vice versa. For $d=2.65 \AA$, a significant direct $\operatorname{Ir}_{1}-\mathrm{Ir}_{2} d$-orbital hybridization is observed. We find this hybridization increasing up to $25 \%$ for $d=2.45 \AA$, resulting in a large bonding-antibonding energy separation, as seen in the corresponding orbital plots in Fig. 3(d). Note that for $d=2.73 \AA$ orbitals with a predominantly $\operatorname{Ir}_{1}$ character are at higher energies than those of $\operatorname{Ir}_{2}$ character, reflecting different on-site orbital energies. This is a direct consequence of the difference in the valence configurations of $\operatorname{Ir}_{1}$ and $\operatorname{Ir}_{2}$ ions and the $\operatorname{Ir}_{1,2} 5 d-\mathrm{O} 2 p$ hybridization.

The effect of the low CF symmetry at two different Ir ions can be estimated by computing the $t_{2 g}$ splittings $\delta$ at each of the Ir ions from restricted active space (RAS) [48] calculations where the $d$ orbital occupation at the other Ir ion is constrained. We find considerably large $t_{2 g}$ splittings of $\delta_{1}=0.58$ and $\delta_{2}=0.60 \mathrm{eV}$ for $\mathrm{Ir}_{1}$ and $\mathrm{Ir}_{2}$, respectively. Such large values compete with the SOC strength of $\sim 0.5 \mathrm{eV}$ of the Ir ions to considerably reduce the effect of SOC, thus resulting in the modification of the local spin-orbit multiplet structure.
The scalar-relativistic ground state realized in $\mathrm{Ba}_{5} \mathrm{AlIr}_{2} \mathrm{O}_{11}$ is the double-exchange ${ }^{4} A_{1 g}$ multiplet [49], an orbitally nondegenerate high-spin quartet, with wave function ${ }^{4} \psi_{0}=$ $\alpha\left|d_{1}^{4}, d_{2}^{5}\right\rangle+\beta\left|d_{1}^{3}, d_{2}^{6}\right\rangle+\gamma\left|d_{1}^{5}, d_{2}^{4}\right\rangle$ with $\alpha^{2}=0.89, \quad \beta^{2}=$ 0.09 , and $\gamma^{2}=0.02$, where $d_{i}^{n}$ corresponds to $n$ electrons in $\operatorname{Ir}_{i} d$ orbitals. The lowest ${ }^{2} T_{1}$ doublet state is $40 \mathrm{meV}$ higher with wave-function weights $\alpha^{2}=0.85, \beta^{2}=0.11$, and $\gamma^{2}=0.02$. We further find that the weight of the $\left|d_{1}^{4}, d_{2}^{5}\right\rangle$ configuration in all the excited multiplet wave functions is greater than $95 \%$. It is interesting to note that excluding all the configurations involving the hopping of electrons from $\mathrm{Ir}_{1}$ to $\mathrm{Ir}_{2}$ and vice versa in the wave function preserve the spin-orbit spectrum except for an overall shift $\leqslant 50 \mathrm{meV}$. The doubleexchange ground state as well as the dominant contribution of the $\left|d_{1}^{4}, d_{2}^{5}\right\rangle$ configuration imply charge separation within the dimer units. Further, the natural orbital occupations obtained from the CASSCF calculations are close to 4 and 5 for $\operatorname{Ir}_{1}$ and $\mathrm{Ir}_{2}$ ions, respectively. Thus, we conclude that the two Ir ions in $\mathrm{Ba}_{5} \mathrm{AlIr}_{2} \mathrm{O}_{11}$ host different ionic states- $\mathrm{Ir}_{1}^{5+}$ and $\mathrm{Ir}_{2}^{4+}$ - which results in charge ordering within the dimers and the low-energy excitations are strictly local to individual Ir ions and not among dimer orbitals.

The SOC results in the admixture of all the 15 nonrelativistic multiplet terms shown in Table I. The addition of angular momenta of two $l_{\text {eff }}=1\left(l_{\text {eff }, 1}=l_{\text {eff, } 2}=1\right)$ sites with spins $s_{1}=1 / 2$ and $s_{2}=1$ gives rise to 84 effective total angular momentum $\left(J_{\text {eff }}\right)$ states. In $\mathrm{Ba}_{5} \mathrm{AlIr}_{2} \mathrm{O}_{11}$, due to the noncubic $\mathrm{CFs}$, all degeneracies are removed except for the Kramers doublet degeneracy. From the analysis of the wave functions, we assign the peaks $A-C$ to excitations from the $\operatorname{Ir}_{1} J_{\text {eff }}=0$ to $J_{\text {eff }}=1$ states. Peak $F$ and satellite feature $H$ consist of excitations from $\operatorname{Ir}_{1} J_{\text {eff }}=0$ to $J_{\text {eff }}=2$ and $\operatorname{Ir}_{2} J_{\text {eff }}=1 / 2$ to $J_{\text {eff }}=3 / 2$ states that are split due to noncubic CFs. Peak $G$ originates from excitations involving $\operatorname{Ir}_{1} J_{\text {eff }}=0$ and $J_{\text {eff }}=2$ states as well. Peaks $I-K$ are the result of simultaneous on-site excitations at $\mathrm{Ir}_{1}$ and $\mathrm{Ir}_{2}$ ions (see Fig. S5 in SM [24]).

At first sight, an insignificant Ir-Ir intradimer $d$-orbital hybridization in $\mathrm{Ba}_{5} \mathrm{AlIr}_{2} \mathrm{O}_{11}$ might be surprising, even though the distance between the Ir sites is close to that of Ir metal. However, due to the crystallographic inequivalence of the two Ir ions in the dimer unit and the different $\mathrm{O}_{6}$ arrangement, the symmetry of split $t_{2 g}$ orbitals at each of the Ir ions is very different and subsequently little direct overlap is realized. In fact, for dimer systems with structurally equivalent ions such as $\mathrm{Ba}_{3} \mathrm{InIr}_{2} \mathrm{O}_{9}$ [7], we find a considerable hybridization resulting in delocalized dimer orbitals [50]. It would be interesting to characterize the local electronic structure in other face-sharing Ir dimer compounds such as $\mathrm{Ba}_{3} \mathrm{ZnIr}_{2} \mathrm{O}_{9}$ and $\mathrm{Ba}_{3} \mathrm{ZrIr}_{2} \mathrm{O}_{9}$ [51] where the Ir dimer unit occupancy is eight and ten, respectively.

\section{CONCLUSION}

In conclusion, we have measured both $\mathrm{Ir} L_{3}-$ and $\mathrm{O}$ $K$-edge RIXS spectra and observed multiple spin-orbital excitations. Our $a b$ initio QC calculations reproduce very well the excitation spectrum up to $3.5 \mathrm{eV}$ observed in the RIXS measurements. We find charge ordering within the Ir dimers with $\operatorname{Ir}_{1}{ }^{5+}\left(d^{4}\right)$ and $\operatorname{Ir}_{2}{ }^{4+}\left(d^{5}\right)$ configurations. We have established a direct connection between the excitations in $\mathrm{Ir}_{2} \mathrm{O}_{9}$ 
dimer units and those at individual Ir ions. The appearance of multiple peaks is a direct consequence of strong noncubic $\mathrm{CFs}$ originating from the distorted octahedral environment around the Ir ions. In spite of the small intradimer Ir-Ir distance, the direct $d-d$ hybridization is relatively weak and the bonding-antibonding splitting is negligible compared to the noncubic CF splittings. Alternatively, we find increased intradimer configuration mixing due to strong electron-electron interactions, particularly the $\left|d_{1}^{3}, d_{2}^{6}\right\rangle$ configuration stabilizing the ground state. This strongly supports nearly complete charge ordering within the Ir dimers in $\mathrm{Ba}_{5} \mathrm{AlIr}_{2} \mathrm{O}_{11}$ and refutes the suggested formation of dimer orbitals [21]. Our results highlight the importance of minute details of the crystal structure to understand the electronic and magnetic properties of clustered iridates and TM magnets in general and calls for reinvestigating several already studied materials with accurate $a b$ initio many-body calculations. Finally, we emphasize that the combination of RIXS and quantum chemistry calculations is an excellent tool to unambiguously decipher complicated electronic structures.

\section{ACKNOWLEDGMENTS}

V.M.K. and O.V. would like to acknowledge funding from Swiss NSF NCCR MARVEL and the Sinergia grant NanoSkyrmionics CR-SII5 171003. The work at PSI is supported by the Swiss NSF through the NCCR MARVEL and the Sinergia network Mott Physics Beyond the Heisenberg Model (MPBH) (SNSF Research Grants No. CRSII2 160765/1 and No. CRSII2 141962). X.L. acknowledges financial support from the European Community's Seventh Framework Programme (FP7/20072013) under Grant Agreement No. 290605 (Cofund; PSI-Fellow). The work at BNU is supported by the National Natural Science Foundation of China under Grants No. 11734002 and No. 11922402. M.D. was partially funded by the Swiss National Science Foundation within the D-A-CH programme (SNSF Research Grant No. 200021L 141325). This research used resources of the Advanced Photon Source, a U.S. Department of Energy (DOE) Office of Science User Facility operated for the DOE Office of Science by Argonne National Laboratory under Contract No. DE-AC02-06CH11357.
[1] M. Imada, A. Fujimori, and Y. Tokura, Metal-insulator transitions, Rev. Mod. Phys. 70, 1039 (1998).

[2] H. F. Pen, J. van den Brink, D. I. Khomskii, and G. A. Sawatzky, Orbital Ordering in a Two-Dimensional Triangular Lattice, Phys. Rev. Lett. 78, 1323 (1997).

[3] D. I. Khomskii and T. Mizokawa, Orbitally Induced Peierls State in Spinels, Phys. Rev. Lett. 94, 156402 (2005).

[4] R. Peierls, More Surprises in Theoretical Physics (Princeton University Press, Princeton, NJ, 1991).

[5] I. Terasaki, S. Ito, T. Igarashi, S. Asai, H. Taniguchi, R. Okazaki, Y. Yasui, K. Kobayashi, R. Kumai, H. Nakao, and Y. Murakami, Novel charge ordering in the trimer iridium oxide $\mathrm{BaIrO}_{3}$, Crystals 6, 27 (2016).

[6] A. Nag, S. Middey, S. Bhowal, S. K. Panda, R. Mathieu, J. C. Orain, F. Bert, P. Mendels, P. G. Freeman, M. Mansson, H. M. Ronnow, M. Telling, P. K. Biswas, D. Sheptyakov, S. D. Kaushik, V. Siruguri, C. Meneghini, D. D. Sarma, I. Dasgupta, and S. Ray, Origin of the Spin-Orbital Liquid State in a Nearly $J=0$ Iridate $\mathrm{Ba}_{3} \mathrm{ZnIr}_{2} \mathrm{O}_{9}$, Phys. Rev. Lett. 116, 097205 (2016).

[7] T. Dey, M. Majumder, J. C. Orain, A. Senyshyn, M. PrinzZwick, S. Bachus, Y. Tokiwa, F. Bert, P. Khuntia, N. Büttgen, A. A. Tsirlin, and P. Gegenwart, Persistent low-temperature spin dynamics in the mixed-valence iridate $\mathrm{Ba}_{3} \mathrm{InIr}_{2} \mathrm{O}_{9}$, Phys. Rev. B 96, 174411 (2017).

[8] A. Nag, S. Bhowal, F. Bert, A. D. Hillier, M. Itoh, I. Carlomagno, C. Meneghini, T. Sarkar, R. Mathieu, I. Dasgupta, and $\mathrm{S}$. Ray, $\mathrm{Ba}_{3} M \mathrm{Ir}_{2} \mathrm{O}_{9}$ hexagonal perovskites in the light of spin-orbit coupling and local structural distortions, Phys. Rev. B 97, 064408 (2018).

[9] A. Revelli, M. Moretti Sala, G. Monaco, P. Becker, L. Bohatý, M. Hermanns, T. C. Koethe, T. Fröhlich, P. Warzanowski, T. Lorenz, S. V. Streltsov, P. H. M. van Loosdrecht, D. I. Khomskii, J. van den Brink, and M. Grüninger, Resonant inelastic x-ray incarnation of Young's double-slit experiment, Sci. Adv. 5, eaav4020 (2019).
[10] H.-S. Kim, J. Im, M. J. Han, and H. Jin, Spin-orbital entangled molecular $j_{\text {eff }}$ states in lacunar spinel compounds, Nat. Commun. 5, 3988 (2014).

[11] M. Y. Jeong, S. H. Chang, B. H. Kim, J.-H. Sim, A. Said, D. Casa, T. Gog, E. Janod, L. Cario, S. Yunoki, M. J. Han, and J. Kim, Direct experimental observation of the molecular $J_{\text {eff }}=$ $3 / 2$ ground state in the lacunar spinel $\mathrm{GaTa}_{4} \mathrm{Se}_{8}$, Nat. Commun. 8, 782 (2017).

[12] M. J. Park, G. Sim, M. Y. Jeong, A. Mishra, M. J. Han, and S. Lee, Pressure-induced topological superconductivity in the spin-orbit Mott insulator $\mathrm{GaTa}_{4} \mathrm{Se}_{8}$, npj Quantum Mater. 5, 41 (2020).

[13] B. J. Kim, H. Jin, S. J. Moon, J. Y. Kim, B. G. Park, C. S. Leem, J. Yu, T. W. Noh, C. Kim, S. J. Oh, J. H. Park, V. Durairaj, G. Cao, and E. Rotenberg, Novel $J_{\text {eff }}=1 / 2$ Mott State Induced by Relativistic Spin-Orbit Coupling in $\mathrm{Sr}_{2} \mathrm{IrO}_{4}$, Phys. Rev. Lett. 101, 076402 (2008).

[14] B. J. Kim, H. Ohsumi, T. Komesu, S. Sakai, T. Morita, H. Takagi, and T. Arima, Phase-Sensitive Observation of a Spin-Orbital Mott State in $\mathrm{Sr}_{2} \mathrm{IrO}_{4}$, Science 323, 1329 (2009).

[15] V. M. Katukuri, H. Stoll, J. van den Brink, and L. Hozoi, Ab initio determination of excitation energies and magnetic couplings in correlated quasi-two-dimensional iridates, Phys. Rev. B 85, 220402(R) (2012).

[16] M. Moretti Sala, S. Boseggia, D. F. McMorrow, and G. Monaco, Resonant X-Ray Scattering and the $j_{\text {eff }}=1 / 2$ Electronic Ground State in Iridate Perovskites, Phys. Rev. Lett. 112, 026403 (2014).

[17] W. Witczak-Krempa, G. Chen, Y. B. Kim, and L. Balents, Correlated quantum phenomena in the strong spin-orbit regime, Annu. Rev. Condens. Matter Phys. 5, 57 (2014).

[18] A. Abragam and B. Bleaney, Electron Paramagnetic Resonance of Transition Ions (Clarendon Press, Oxford, UK, 1970).

[19] G. Khaliullin, Excitonic Magnetism in Van Vleck Type $d^{4}$ Mott Insulators, Phys. Rev. Lett. 111, 197201 (2013). 
[20] M. Kusch, V. M. Katukuri, N. A. Bogdanov, B. Büchner, T. Dey, D. V. Efremov, J. E. Hamann-Borrero, B. H. Kim, M. Krisch, A. Maljuk, M. M. Sala, S. Wurmehl, G. AslanCansever, M. Sturza, L. Hozoi, J. van den Brink, and J. Geck, Observation of heavy spin-orbit excitons propagating in a nonmagnetic background: The case of $(\mathrm{Ba}, \mathrm{Sr})_{2} \mathrm{YIrO}_{6}$, Phys. Rev. B 97, 064421 (2018).

[21] Y. Wang, R. Wang, J. Kim, M. H. Upton, D. Casa, T. Gog, G. Cao, G. Kotliar, M. P. M. Dean, and X. Liu, Direct Detection of Dimer Orbitals in $\mathrm{Ba}_{5} \mathrm{AlIr}_{2} \mathrm{O}_{11}$, Phys. Rev. Lett. 122, 106401 (2019).

[22] H. k. Müller-Buschbaum and Ch. Lang, $\mathrm{Ba}_{5} \mathrm{AlIr}_{2} \mathrm{O}_{11}$ : Eine neue Verbindung mit Iridium(IV, V), Z. Anorg. Allg. Chem. 568, 29 (1989).

[23] J. Terzic, J. C. Wang, Feng Ye, W. H. Song, S. J. Yuan, S. Aswartham, L. E. DeLong, S. V. Streltsov, D. I. Khomskii, and G. Cao, Coexisting charge and magnetic orders in the dimer-chain iridate $\mathrm{Ba}_{5} \mathrm{AlIr}_{2} \mathrm{O}_{11}$, Phys. Rev. B 91, 235147 (2015).

[24] See Supplemental Material at http://link.aps.org/supplemental/ 10.1103/PhysRevB.105.075114 for additional RIXS plots and computational details.

[25] M. Moretti Sala, K. Martel, C. Henriquet, A. Al Zein, L. Simonelli, Ch. J. Sahle, H. Gonzalez, M.-C. Lagier, C. Ponchut, S. Huotari, R. Verbeni, M. Krisch, and G. Monaco, A high-energy-resolution resonant inelastic X-ray scattering spectrometer at ID20 of the European Synchrotron Radiation Facility, J. Synchrotron Radiat. 25, 580 (2018).

[26] J. Kim, D. Casa, M. H. Upton, T. Gog, Y.-J. Kim, J. F. Mitchell, M. van Veenendaal, M. Daghofer, J. van den Brink, G. Khaliullin, and B. J. Kim, Magnetic Excitation Spectra of $\mathrm{Sr}_{2} \mathrm{IrO}_{4}$ Probed by Resonant Inelastic X-Ray Scattering: Establishing Links to Cuprate Superconductors, Phys. Rev. Lett. 108, 177003 (2012)

[27] V. N. Strocov, T. Schmitt, U. Flechsig, T. Schmidt, A. Imhof, Q. Chen, J. Raabe, R. Betemps, D. Zimoch, J. Krempasky, X. Wang, M. Grioni, A. Piazzalunga, and L. Patthey, Highresolution soft X-ray beamline ADRESS at the Swiss Light Source for resonant inelastic X-ray scattering and angleresolved photoelectron spectroscopies, J. Synchrotron Radiat. 17, 631 (2010).

[28] G. Ghiringhelli, A. Piazzalunga, C. Dallera, G. Trezzi, L. Braicovich, T. Schmitt, V. N. Strocov, R. Betemps, L. Patthey, $\mathrm{X}$. Wang, and M. Grioni, SAXES, a high resolution spectrometer for resonant $\mathrm{x}$-ray emission in the $400-1600 \mathrm{eV}$ energy range, Rev. Sci. Instrum. 77, 113108 (2006).

[29] X. Lu, P. Olalde-Velasco, Y. Huang, V. Bisogni, J. Pelliciari, S. Fatale, M. Dantz, J. G. Vale, E. C. Hunter, J. Chang, V. N. Strocov, R. S. Perry, M. Grioni, D. F. McMorrow, H. M. Rønnow, and T. Schmitt, Dispersive magnetic and electronic excitations in iridate perovskites probed by oxygen $K$-edge resonant inelastic x-ray scattering, Phys. Rev. B 97, 041102(R) (2018)

[30] T. Helgaker, P. Jørgensen, and J. Olsen, Molecular ElectronicStructure Theory (Wiley, Chichester, UK, 2000).

[31] F. Neese, The ORCA program system, WIREs Comput. Mol. Sci. 2, 73 (2012).

[32] M. Klintenberg, S.E. Derenzo, and M.J. Weber, Accurate crystal fields for embedded cluster calculations, Comput. Phys. Commun. 131, 120 (2000).
[33] D. A. Pantazis, X.-Y. Chen, C. R. Landis, and F. Neese, All-electron scalar relativistic basis sets for third-row transition metal atoms, J. Chem. Theory Comput. 4, 908 (2008).

[34] T. H. Dunning, Gaussian basis sets for use in correlated molecular calculations. I. The atoms boron through neon and hydrogen, J. Chem. Phys. 90, 1007 (1989).

[35] A. Bergner, M. Dolg, W. Küchle, H. Stoll, and H. Preuß, Ab initio energy-adjusted pseudopotentials for elements of groups 13-17, Mol. Phys. 80, 1431 (1993).

[36] P. Fuentealba, L. von Szentpaly, H. Preuss, and H. Stoll, Pseudopotential calculations for alkaline-earth atoms, J. Phys. B 18, 1287 (1985).

[37] C. Angeli, R. Cimiraglia, and J.-P. Malrieu, $N$-electron valence state perturbation theory: A fast implementation of the strongly contracted variant, Chem. Phys. Lett. 350, 297 (2001).

[38] J. Kim, M. Daghofer, A. H. Said, T. Gog, J. van den Brink, G. Khaliullin, and B. J. Kim, Excitonic quasiparticles in a spinorbit Mott insulator, Nat. Commun. 5, 4453 (2014).

[39] E. Paris, Y. Tseng, E. M. Pärschke, W. Zhang, M. H. Upton, A. Efimenko, K. Rolfs, D. E. McNally, L. Maurel, M. Naamneh, M. Caputo, V. N. Strocov, Z. Wang, D. Casa, C. W. Schneider, E. Pomjakushina, K. Wohlfeld, M. Radovic, and T. Schmitt, Strain engineering of the charge and spin-orbital interactions in $\mathrm{Sr}_{2} \mathrm{IrO}_{4}$, Proc. Natl. Acad. Sci. USA 117, 24764 (2020).

[40] H. Gretarsson, J. P. Clancy, X. Liu, J. P. Hill, E. Bozin, Y. Singh, S. Manni, P. Gegenwart, J. Kim, A. H. Said, D. Casa, T. Gog, M. H. Upton, H.-S. Kim, J. Yu, V. M. Katukuri, L. Hozoi, J. van den Brink, and Y.-J. Kim, Crystal-Field Splitting and Correlation Effect on the Electronic Structure of $A_{2} \mathrm{IrO}_{3}$, Phys. Rev. Lett. 110, 076402 (2013).

[41] To simulate electron-hole exciton peaks one would need to calculate $N-1$ (removal of an electron) state calculations.

[42] V. M. Katukuri, K. Roszeitis, V. Yushankhai, A. Mitrushchenkov, H. Stoll, M. van Veenendaal, P. Fulde, J. van den Brink, and L. Hozoi, Electronic structure of low-dimensional $4 d^{5}$ oxides: Interplay of ligand distortions, overall lattice anisotropy, and spin-orbit interactions, Inorg. Chem. 53, 4833 (2014).

[43] See direct product tables for $O_{h}$ point group representations, e.g., in Ref. [52].

[44] For example, for pure trigonal distortions, the $t_{2 g}$ orbitals are split into $a_{1 g}$ and $e_{g}$.

[45] S. V. Streltsov and D. I. Khomskii, Covalent bonds against magnetism in transition metal compounds, Proc. Natl. Acad. Sci. USA 113, 10491 (2016).

[46] The canonical orbitals are eigenfunctions of the so-called (effective single particle) Fock operator.

[47] The orbital compositions are obtained from the Löwdin natural orbital decomposition.

[48] P. A. Malmqvist, A. Rendell, and B. O. Roos, The restricted active space self-consistent-field method, implemented with a split graph unitary group approach, J. Phys. Chem. 94, 5477 (1990).

[49] S. V. Streltsov, G. Cao, and D. I. Khomskii, Suppression of magnetism in $\mathrm{Ba}_{5} \mathrm{AlIr}_{2} \mathrm{O}_{11}$ : Interplay of Hund's coupling, molecular orbitals, and spin-orbit interaction, Phys. Rev. B 96, 014434 (2017). 
[50] V. M. Katukuri and O. V. Yazyev, Delocalized dimer orbitals in $\mathrm{Ba}_{3} \mathrm{InIr}_{2} \mathrm{O}_{9}$ (to be published).

[51] T. Sakamoto, Y. Doi, and Y. Hinatsu, Crystal structures and magnetic properties of $6 H$-perovskite-type oxides $\mathrm{Ba}_{3} \mathrm{MIr}_{2} \mathrm{O}_{9}$
$(M=\mathrm{Mg}, \mathrm{Ca}, \mathrm{Sc}, \mathrm{Ti}, \mathrm{Zn}, \mathrm{Sr}, \mathrm{Zr}, \mathrm{Cd}$ and In), J. Solid State Chem. 179, 2595 (2006).

[52] P. W. Atkins, M. S. Child, and C. S. G. Phillips, Tables for Group Theory (Oxford University Press, London, 1993). 